\title{
LES Simulation of Oscillating Natural Ventilation Driven by Vortex Shedding in Isolated Buildings
}

\author{
Daniel Albuquerque ${ }^{1}$, Mats Sandberg ${ }^{2}$, Paul Linden ${ }^{3}$, Guilherme Carrilho da Graça ${ }^{1}$ \\ ${ }^{1}$ Instituto Dom Luiz, Faculdade de Ciências, Universidade de Lisboa, Lisboa, Portugal \\ ${ }^{2}$ Department of Building, Energy and Environmental Engineering, University of Gävle, Gävle, \\ Sweden \\ ${ }^{3}$ Department of Applied Mathematics and Theoretical Physics University of Cambridge, \\ Cambridge, United Kingdom
}

\begin{abstract}
A recently published study presented a new type of natural ventilation (NV) flow, named pumping ventilation. The oscilatory mechanism of vortex shedding that occurs at the wake region of an isolated building drives this new type of ventilation in rooms with two (or more) openings facing the leeward or windward side of an isolated building.

This paper presents a validated Large Eddy Simulation (LES) study of oscillating/pumping NV in an isolated building using three different separations (s') between its two windows. LES is validated using an experimental database from measurements performed at the University of Gävle boundary layer wind tunnel (WT). The measurements use a cubic model with $0.45 \mathrm{~m}$ side representing a three-story building at a 1/20 scale that allows the use of bottom-hung windows. LES results show a good agreement with the measured nondimensional ventilation rates. A dimensionless analysis shows the dominant frequencies of the pumping flow, are close to the Strouhal frequency.
\end{abstract}

\section{Introduction}

From a fluid mechanics perspective, buildings can be regarded as bluff stationary bodies obstructing the wind. This obstruction creates flow boundary layers near the front and sides of the building, that, in the downstream region of the flow separate from the building surfaces to create a wake surrounded by two shear layers. The interaction of these shear layers creates periodic vortex shedding that may drive airflow in rooms with two (or more) openings facing the leeward or windward side of the building. This recently discovered (Daish et al. 2016) type of natural ventilation flow is called pumping ventilation, in reference to the alternating nature of the shear layer instability that drives the flow. The finding was made possible by an extensive WT measurements campaign that covered different natural ventilation strategies and whose results were the basis of guidelines for building designers (Linden et al. 2016).

As an approach to study natural ventilation, WT measurements are known for their accuracy and ease of use. However, WT studies may also have several limitations, e.g. the size of the models is often restricted due to the wind blockage effects; or, regarding the measuring equipment, being difficult to measure the correct variable at the crucial locations. To facilitate the analysis, computational fluids dynamics (CFD), can be used to calculate a flow solution, working in combination with WT studies.

\section{Brief review on CFD simulations for natural ventilation on buildings}

CFD has been increasingly used as a tool to study flow patterns around buildings and natural ventilation driving mechanisms (Blocken 2014). Despite the unsteadiness that defines every natural ventilation flow, there are some, such as single-sided ventilation through one aperture (SS1), cross-ventilation between two opposite façades (CV) and corner-ventilation between adjacent façades (CR), where their ventilation driving forces may be considered as quasi-stationary since the flow is statistically steady (Linden 1999). Therefore, for these ventilation mechanisms, RANS may be a suitable choice due to its ease of implementation, faster computation time and reliable results. RANS has another great advantage over other turbulence models such as LES, hybrids RANS-LES or DNS; it has been successfully applied and validated for different flow mechanisms during the past decades (Shirzadi, Naghashzadegan, and A. Mirzaei 2018; Park et al. 2017; Linden et al. 2016). However, there are indications, from previous studies (Blocken 2015; Yoder, DeBonis, and Georgiadis 2015; Ricci et al. 2018), that RANS CFD models may not be a suitable approach for flows driven by unsteady shear such as pumping ventilation. For these cases LES may be a more suitable approach that, in combination with WT experiments, can be used to investigate the flow driving mechanism behind pumping NV.

Kobayashi et. al (2018) performed a CFD study of a threedimensional (3D) cubic model with two plain apertures, using LES. The study includes three cases of pumping ventilation with variable window separation, s'. The results show there is an increase in the ventilation rate for higher separations (confirming the results obtained by Daish et al. 2016). Finally, it concludes the empirical orifice equation has limitations when the wind pressure coefficient difference (WPCD) is below 0.1. Furthermore, a new empirical model, based on the orifice equation, is proposed with a new parameter addition: the standard deviation of WPCD. The estimated airflow rate error for pumping ventilation is always below $30 \%$. 

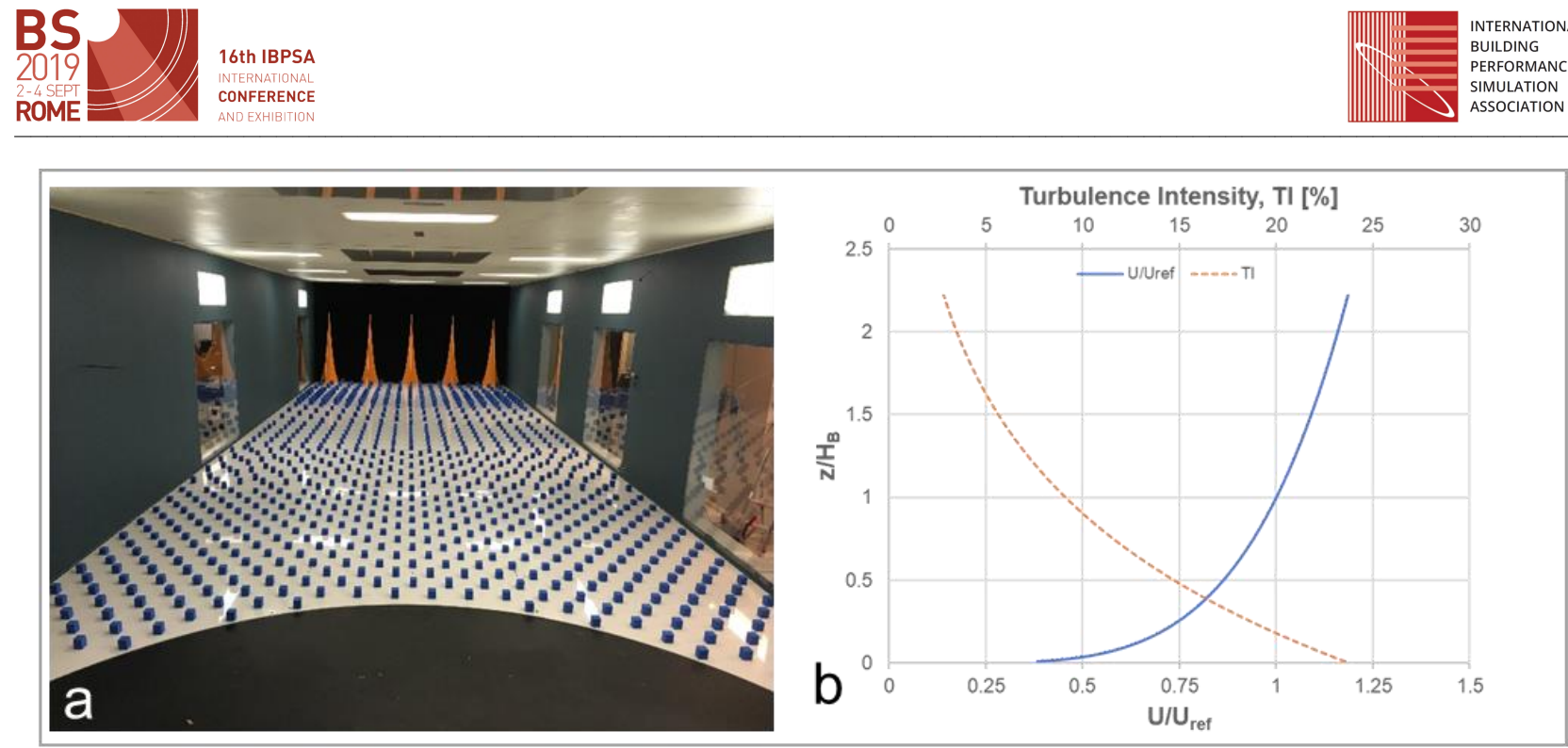

Figure 1: (a) Distribution of spires and roughness elements within the wind tunnel; (b) Wind velocity and turbulence intensity measured profiles of the WT boundary layer.

\section{Present study}

This paper presents LES simulations of oscillating NV driven by vortex shedding in an isolated building, validating LES with a high-quality WT experimental dataset. The model used in the WT experiments is more realistic than typical studies due to the inclusion of a real type of window, being of the utmost importance since most real buildings do not have plain openings; instead, a partially opened and tilted windows are the usual NV configuration. After validation, the LES model is used to investigate the strength of the flow driving mechanism, that, in a previous study (Daish et al. 2016; Kobayashi et al. 2018), was found to increase with opening separation (s) scaled by the building width $\left(\mathrm{W}_{\mathrm{b}}\right), \mathrm{s}=\mathrm{s} / \mathrm{W}_{\mathrm{b}}$.

\section{Wind tunnel experiments}

\section{Wind tunnel description}

The experiments of this study are performed at the atmospheric boundary layer wind tunnel of Gävle University (HiG), in Gävle, Sweden. This closed-circuit wind tunnel has a test section of $3 \mathrm{~m}$ [width] by $1.5 \mathrm{~m}$ [height] that extends by $11 \mathrm{~m}$ [length] (see Figure 1-a). The WT airflow is provided by two large fans that generate air velocities between $0.5 \mathrm{~m} / \mathrm{s}$ and $27 \mathrm{~m} / \mathrm{s}$ at the turntable center. Inside the WT, air temperature is controlled through a thermocouple and an HVAC system is available to maintain constant a predetermined air temperature. As shown in Figure 1-a, during the measurements the WT has a set of five equally spaced triangular spires placed at the inlet of the test section and parallelepiped shaped surface roughness elements situated at the ground from the spires to the turntable, creating the intended boundary layer at the measurement site. Figure 1-b shows the wind velocity and turbulence intensity (TI) profiles of the given boundary layer.

\section{Experimental measurements}

The three-story model used for the WT experiments is a reduced-scale cubic building with a $0.45 \mathrm{~m}$ side, representing a 1/20 scaled building (see Figure 2). Each floor has $0.15 \mathrm{~m}$ height. Measurements were performed only on the middle floor. The measured room has $4 \mathrm{~mm}$ thickness walls and the bottom-hung square-window aperture has $0.075 \mathrm{~m}$ side; is tilted by 14 degrees to the interior of the room; and it has the same thickness of the walls. The measurements are made for three different window separations, s': $0.25,0.50$ and 0.75 . The ventilation rate is measured using the decay method. Carbon-dioxide $\left(\mathrm{CO}_{2}\right)$ is used as a tracer gas, injected from the floor of the room, close to its center using a thin vertical rod with holes in various directions (achieving well-mixed conditions). A sampling grid with 15 evenly spaced points is used to measure the $\mathrm{CO}_{2}$ concentration levels inside the room at a $20 \mathrm{~Hz}$ rate. The use of the decay method to measure effective flow, combined with a closed WT make the measuring of the background $\mathrm{CO}_{2}$ concentration mandatory. Therefore, the $\mathrm{CO}_{2}$ concentration inside the WT is also measured.

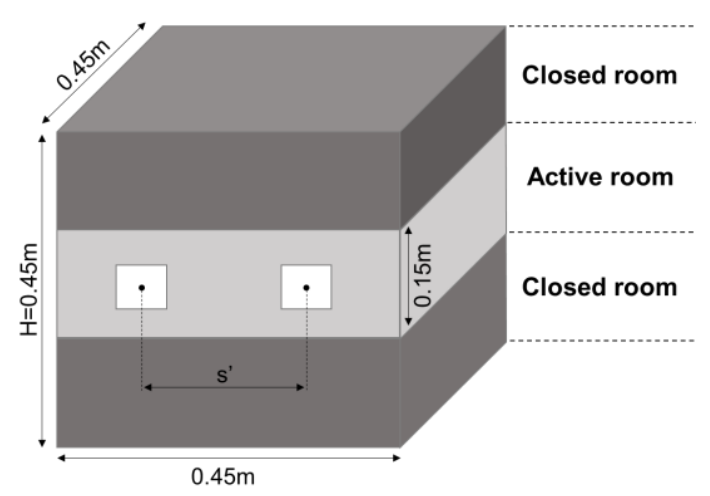

Figure 2: Dimensions of the scaled building model used in the wind tunnel measurements.

\section{Experimental results}

Figure 3-a shows the background and the interior mean $\mathrm{CO}_{2}$ concentrations during a WT pumping ventilation measurement for the case with a separation of 0.50 . The shape of this $\mathrm{CO} 2$ concentration is similar across all the measured cases, the typical shape of a pollutant concentration when a decay method is used to measure the ventilation rate. Initially, there is a period of 

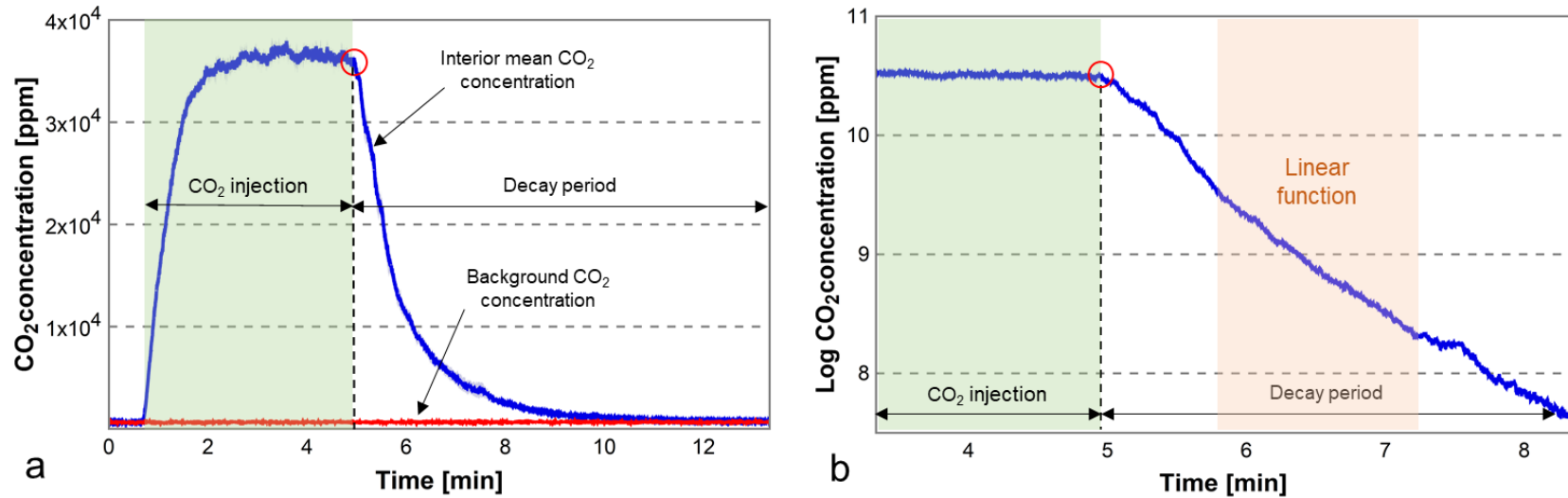

Figure 3: (a) Interior mean and background $\mathrm{CO}_{2}$ concentration profiles during a measurement; (b) Logarithm of the interior mean $\mathrm{CO}_{2}$ concentration and the linear period during the decay.

pollutant injection and mixing inside the measured room, resulting in a great increase of $\mathrm{CO}_{2}$ concentration inside the room. The next period is when the pollutant concentration stabilizes. Finally, last period is when the pollutant injection stops, and its concentration starts decaying with the non-polluted air that flows in. Calculating the ventilation rate through a multi-point method requires that the logarithmic $\mathrm{CO}_{2}$ concentration during the decay period behaves as a linear function, as shown in Figure 3-b.

To compare the three measured cases a normalized ventilation rate, $Q^{\prime}$, is used

$$
Q^{\prime}=Q /\left(U_{\text {ref }} \cdot A_{\text {eff }}\right)
$$

Where $Q$ is the ventilation rate in $\mathrm{m}^{3} / \mathrm{s}$; and $U_{\text {ref }}$ is the reference velocity which in this study is the velocity at building's height $U_{H}(\approx 3.5 \mathrm{~m} / \mathrm{s})$. $A_{\text {eff }}$ is the effective opening area, defined by

$$
A_{\text {eff }}=\left(A_{1} \cdot A_{2}\right) /\left(A_{l}^{2}+A_{2}^{2}\right)^{1 / 2}
$$

Where $A_{1}$ and $A_{2}$ are the area of each opening $\left[\mathrm{m}^{2}\right]$.

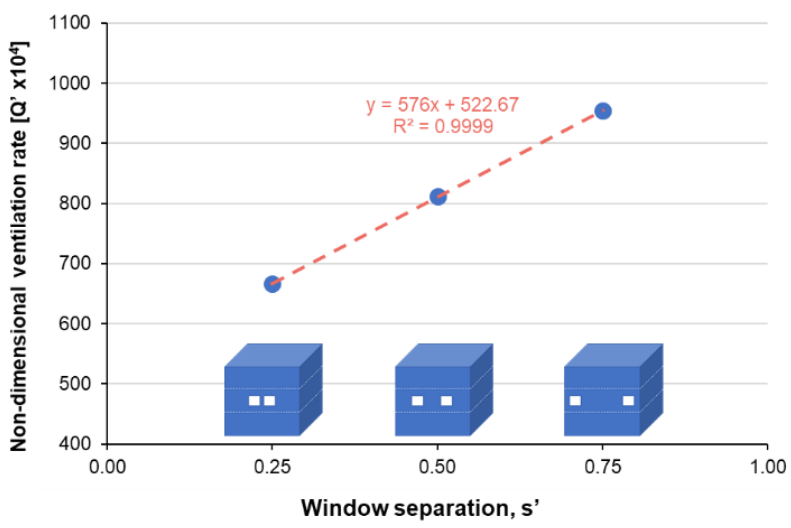

Figure 4: The three measured ventilation rates with a linear trendline and its correlation coefficient, $R^{2}$.

The variation of the non-dimensional ventilation rate, Q', with the window separation, s', is shown on Figure 4. The non-dimensional ventilation rate increases linearly with $\mathrm{s}$, as the correlation coefficient, $\mathrm{R}^{2}$, very close to one suggests. The ventilation improvement is notorious; it goes from a value of 666 when s' $=0.25$ to a value of 954 when $\mathrm{s}^{\prime}=0.75$, an increase of $43 \%$.
As can be seen on Figure 5, three pumping ventilation crucial moments are captured. Injecting smoke inside the measured room with the WT in running conditions, allows for a perfect visualization of this driving mechanism: the vortex shedding. On Figure 5-a there is a vortex close to the right trailing edge of the building that creates a highpressure zone at the right window. Thus, the air flows in from the right window and flows out from the left window, as the smoke suggests. On Figure 5-b stands for the transition period between the right vortex that is weakening and going downstream, and the left vortex that is taking shape by the instability created upstream at the building's front façade. Lastly, on Figure 5-c the left vortex is already formed, creating a high-pressure zone close to the left window (inflow) and a low-pressure zone at the right window (outflow).

\section{CFD simulation}

The CFD simulations were performed using the commercial package software of ANSYS Fluent 19.2. The three measured cases from the WT are simulated and validated using a single meshing method.

\section{Computational domain and mesh}

The CFD model has the same scale as the WT model and the computational domain dimensions are the recommended by the CFD best practice guidelines (Franke 2007). Therefore, the top of the domain is put $5 \mathrm{H}$ away from the top of the model, as well as the side boundaries and the inlet. Finally, an outlet is located at $15 \mathrm{H}$ from the back of the model. As shown in Figure 6-a, the total dimensions of the domain are $4.95(\mathrm{~W}) \times 9.45(\mathrm{~L})$ $\mathrm{x} 2.70(\mathrm{H}) \mathrm{m}^{3}$.

The mesh used for these CFD simulations is based on a mixture of different types of cells, resulting from the new ANSYS Fluent mesh feature called Poly-Hexcore (see Figure 6-b). The feature produces non-tetrahedral meshes; mixing prismatic, polyhedral and hexahedral cells together to avoid highly skewed cells that are often produced in meshes with tetrahedral cells. The meshes have a minimum volume of $1.2 \times 10^{-11} \mathrm{~m}^{3}$ and a maximum volume cell of $2.1 \times 10^{-3} \mathrm{~m}^{3}$. With more than 5000 cells in the cross section of each window and hundreds of thousands of cells in the downstream vicinity of the 


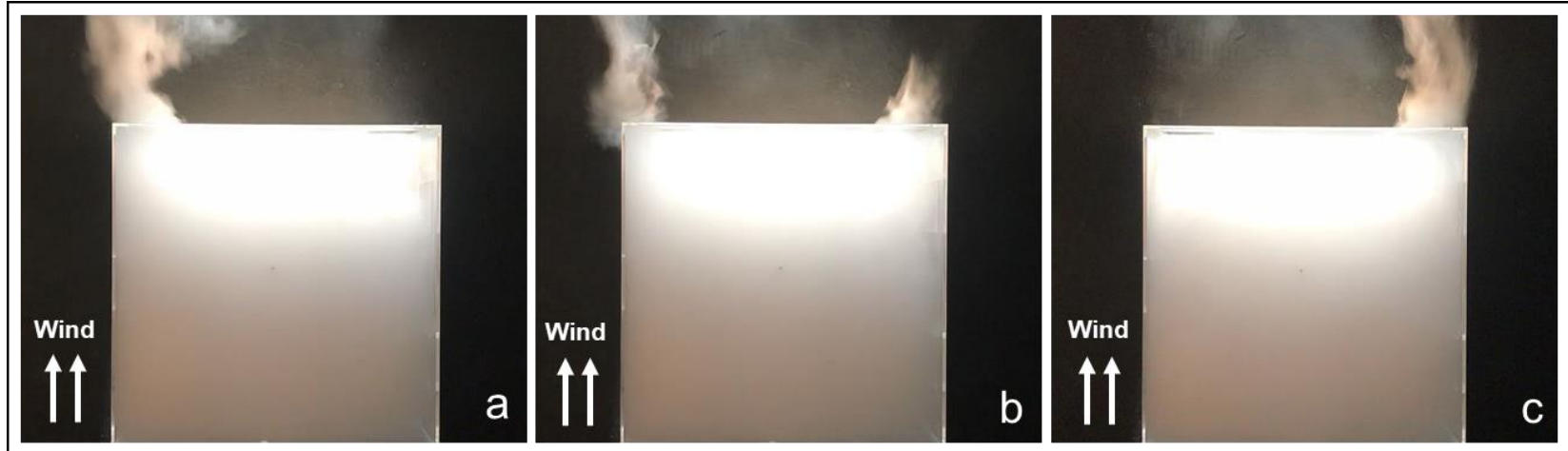

Figure 5: Top view of the oscillatory driving mechanism for pumping ventilation using smoke (s' $=0.75)$ : (a) presence of

a left vortex - air flows from the right window to the left window; $(b)$ no presence of vortexes - transition period between the left and right vortexes (and vice versa); (c) presence of the right vortex - air flows from the left window to the right window.
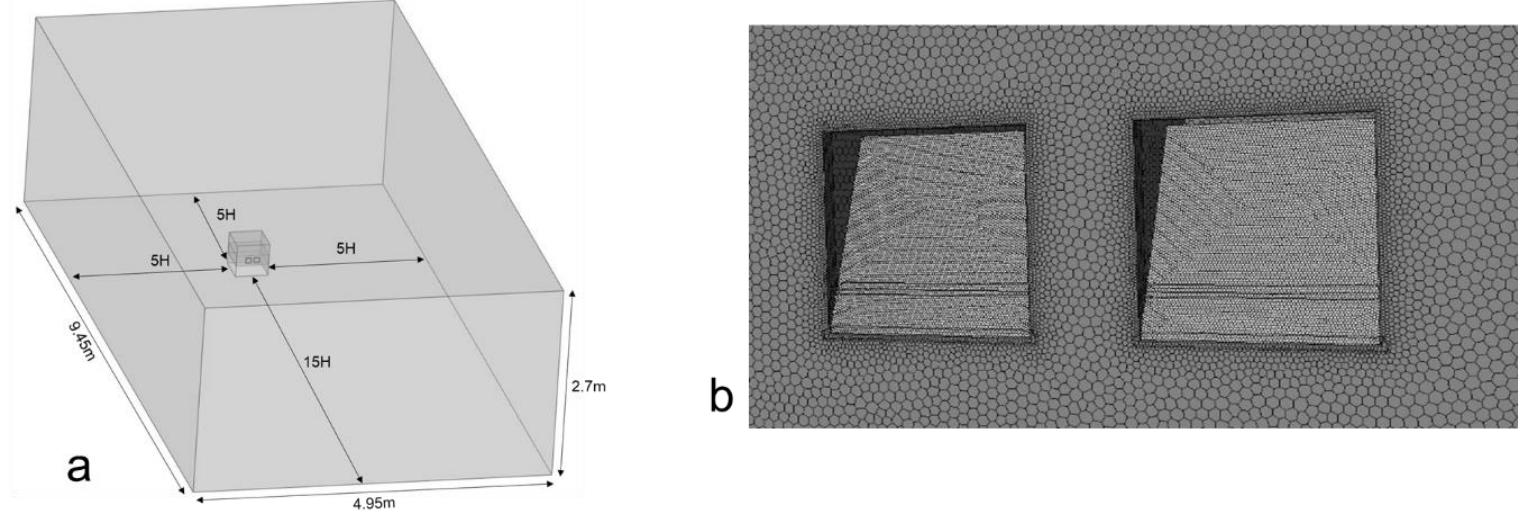

Figure 6: (a) Domain dimensions; (b) Close view of the window's mesh for a separation s' $=0.25$.

Table 1: Number of cells for each LES simulation.

\begin{tabular}{|c|c|}
\hline Window separation, $\mathbf{s}$ & Number of cells \\
\hline 0.25 & 1605525 \\
\hline 0.50 & 1716368 \\
\hline 0.75 & 1666202 \\
\hline
\end{tabular}

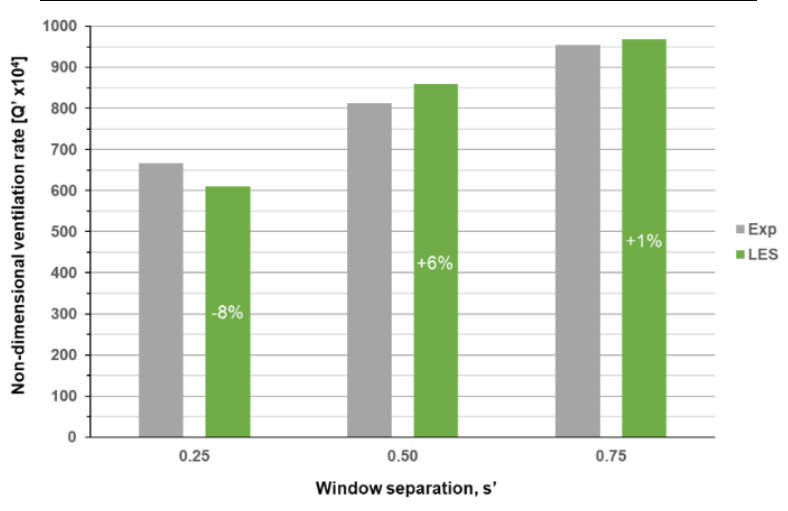

Figure 7: Comparison of non-dimensional ventilation rate between WT experimental measurements and the LES simulations.

model, the pumping mechanism can be properly solved at the windows as well as the vortex shedding mechanism that drives the flow. The growth rate is imposed to be always less than 1.2. Table 1 shows the number of cells for each simulated case.

\section{Boundary conditions}

A uniform velocity distribution across the inlet is imposed in order to have an approaching wind profile (measured with an empty domain) where the computed velocity at building's height, $U_{\mathrm{H}}$, equals the measured velocity at the wind tunnel for the same height. The outlet plane has a zero static pressure condition applied. Both the sides and top boundaries are set to zero normal velocities and zero normal gradients to the remaining flow variables. The model surfaces and the domain ground are defined as stationary walls with the no slip shear condition and have a zero diffusive flux condition for the pollutant specie. A constant volumetric source of $\mathrm{CO} 2$ pollutant is set within the measured room during the simulations with a flux of $8.2 \times 10^{-5} \mathrm{~kg} /\left(\mathrm{m}^{3} . \mathrm{s}\right)$.

\section{Numerical methods}

The inherently transient LES equations are solved using the Smagorinsky-Lilly (SL) turbulence model with a dynamic subgrid-scale (DSGS) model for the filtering operation (Germano et al. 1991). This combination of the SL turbulence model with a DSGS model was recently used to validate SS2 natural ventilation flows (Arinami et al. 2019). For the velocity-pressure coupling, the Pressure-Implicit with Splitting of Operators (PISO) scheme is used in combination the Non-Iterative TimeAdvancement (NITA) scheme. The convection terms have a bounded central differencing schemes and the 

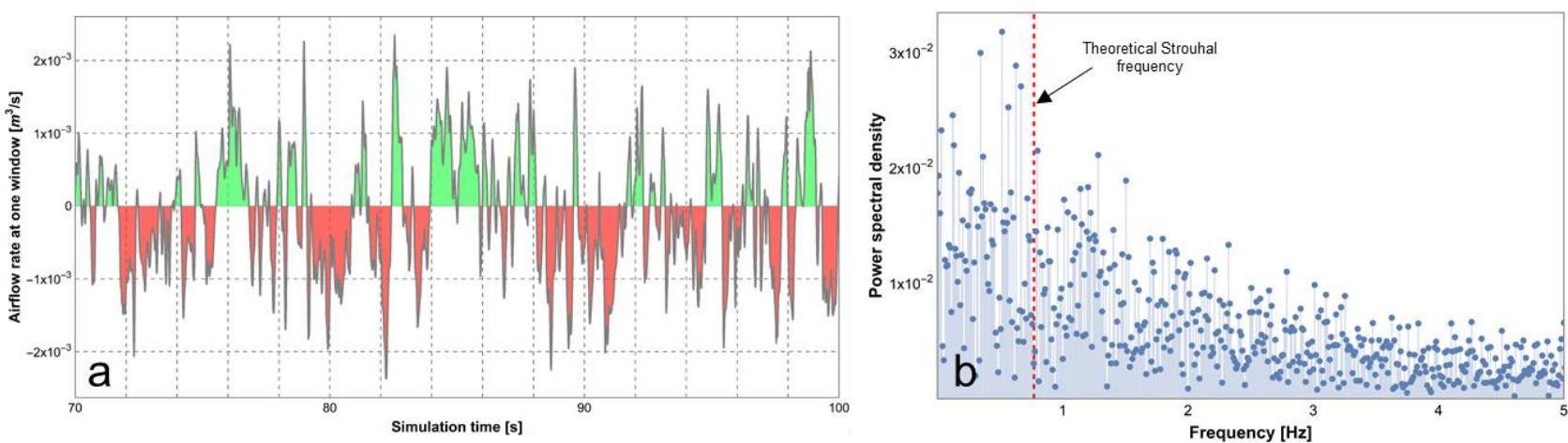

Figure 8: (a) Flow rate at one of the model's window (green: inflow; red: outflow); (b) Power spectra density of the flow rate (red dashed line: theoretical dominant frequency). Both results refer to $s^{\prime}=0.25$.

pressure and $\mathrm{CO}_{2}$ terms have a second order discretization schemes. A bounded second order implicit scheme is set as the transient formulation. The time step size is $1 \times 10^{-3} \mathrm{~s}$, giving an average Courant-Friedrichs-Lewy (CFL) number of 0.36 . The simulations run for 100000 timesteps, resulting in a total of 100 s and more than 36 flow-through times (the entire computational domain). The minimum value for the residuals is identical for the three simulations and are $10^{-6}$ for momentum and $10^{-5}$ for continuity and $\mathrm{CO}_{2}$.

\section{Simulation results}

\section{Ventilation rate comparison}

The method used to extract the effective ventilation rate from the LES simulation was the constant release method. A simulation period of 100 s allowed for the interior mean $\mathrm{CO} 2$ concentration to stabilize and remain constant during several seconds. The non-dimensional ventilation rate, shown in Figure 7, is an average value of the last five seconds of each simulation.

LES results show the three simulated cases have an error lower than $10 \%$ when compared to the experimental measurements in the WT. For the cases s' $=0.25, \mathrm{~s}^{\prime}=0.50$ and $s^{\prime}=0.75$ the errors were $-8 \%,+6 \%$ and $-1 \%$, respectively, as shown in Figure 7.

The LES results are also treated using power spectral analysis. The results from this method were compared with the Strouhal frequency, obtained from the following expression

$$
S_{t}=f_{d} . L / U_{\infty}
$$

In which $f_{d}$ is the dominant frequency of the phenomenon in $\mathrm{Hz}, L$ is the characteristic length of the object [m] and $U_{\infty}$ is the free-stream velocity $[\mathrm{m} / \mathrm{s}]$. A typical $\mathrm{St}$ number for rectangular cylinders is 0.10-0.15 (Knisely 1990). Figure 8-a shows the flow rate through one of the windows of the model during the last 30 simulation seconds. The green and red zones show when that window is the inflow or the outflow region, respectively. Applying a discrete Fourier transform to the simulated flow rate data converts its time domain into a frequency domain. Thus, the dominant frequency of the vortex shedding can be identified. Figure 8-b shows the frequency domain of the simulated window's flow rate where the highest peak of the spectrum is the simulated dominant frequency $\left(f_{d}=\right.$ $0.51 \mathrm{~Hz}$ ). The red dashed line represents the theoretical dominant frequency of the vortex shedding for a typical St number $\left(f_{d}=0.77 \mathrm{~Hz}\right)$.

\section{Conclusion}

This paper presents a study of the oscillatory mechanism that drives pumping natural ventilation in an isolated building with two bottom-hung windows.

Experimental results show that the ventilation rate is proportional to the window separation, s'. The LES simulation is validated for all cases using WT measurements. The simulated ventilation rates are in close agreement with the measured ventilation rates in the WT, showing an error that is always below $10 \%$. LES simulations also show the expected oscillatory flow rate. Further analysis shows a simulated vortex shedding frequency that is $33 \%$ lower that the theoretical value. Although there is a discrepancy between the simulated and theoretical, it is important to point out that the theoretical Strouhal number comes from two-dimensional vortex shedding studies. The pumping flow driving mechanism is three-dimensional.

These preliminary results indicate that LES is suitable to predict with good accuracy the ventilation rate that is driven by a pumping mechanism for an isolated building.

\section{Limitations of present study}

This study may have some limitations related with: a lack of a grid sensitivity study, which may impact the confidence in the obtained results; the use of a uniform velocity distribution across the inlet, where a proper atmospheric boundary layer may be imposed in the future; values of CFL that are higher than 1 within few cells.

\section{Acknowledgements}

The authors gratefully acknowledge the assistance of Leif Claesson during the wind tunnel measurements; and financial support provided by the Fundação para a Ciência e a Tecnologia (Grant No. PD/BD/105995/2014) and Instituto Dom Luiz (UID/GEO/50019/2019).

\section{References}

Arinami, Yuki, Shin-ichi Akabayashi, Yoshihide Tominaga, and Jun Sakaguchi. 2019. "Performance Evaluation of Single-Sided Natural Ventilation for Generic Building Using Large-Eddy Simulations: Effect of Guide Vanes and Adjacent Obstacles." Building and Environment. Elsevier Ltd. 
doi:10.1016/j.buildenv.2019.01.021.

Blocken, Bert. 2014. "Journal of Wind Engineering 50 Years of Computational Wind Engineering: Past, Present and Future \$." Jnl. of Wind Engineering and Industrial Aerodynamics 129. Elsevier: 69-102. doi:10.1016/j.jweia.2014.03.008.

Blocken, Bert. 2015. "Computational Fluid Dynamics for Urban Physics: Importance, Scales, Possibilities, Limitations and Ten Tips and Tricks towards Accurate and Reliable Simulations." Building and Environment 91. Elsevier: 219-245. doi:10.1016/j.buildenv.2015.02.015.

Daish, N. C., G. Carrilho da Graça, P. F. Linden, and D. Banks. 2016. "Impact of Aperture Separation on Wind-Driven Single-Sided Natural Ventilation." Building and Environment 108. Elsevier Ltd: 122134. doi:10.1016/j.buildenv.2016.08.015.

Franke, Jörg. 2007. Best Practice Guideline for the CFD Simulation of Flows in the Urban Environment. Meteorological Inst.

Germano, Massimo, Ugo Piomelli, Parviz Moin, and William H. Cabot. 1991. "A Dynamic SubgridScale Eddy Viscosity Model." Physics of Fluids A 3 (7): 1760-1765. doi:10.1063/1.857955.

Knisely, C. W. 1990. "Strouhal Numbers of Rectangular Cylinders at Incidence: A Review and New Data." Journal of Fluids and Structures 4 (4): 371-393. doi:10.1016/0889-9746(90)90137-T.

Kobayashi, Tomohiro, Mats Sandberg, Takuya Fujita, Eunsu Lim, and Noriko Umemiya. 2018. "SIMPLIFIED ESTIMATION OF WINDINDUCED NATURAL VENTILATION RATE CAUSED BY TURBULENCE FOR A ROOM WITH MINUTE WIND PRESSURE." In PROCEEDINGS - Roomvent \& Ventilation 2018, 613-618.
https://www.lyyti.fi/reg_att/efA658E7F74904eE7 C/D78bE5731da5113EF848.pdf.

Linden, P. F. 1999. "The Fluid Mechanics of Natural Ventilation." Annual Review of Fluid Mechanics 31 (1): 201-238. doi:10.1146/annurev.fluid.31.1.201.

Linden, P. F., K. Adams, E. Arens, D. Banks, S. Brunswick, G. Carrilho da Graca, N. Daish, et al. 2016. "Natural Ventilation for Energy Savings in California Commercial Buildings." Final Project Report, California Energy Commission. June. 516 Pp. http://www.escholarship.org/uc/item/4cd386s7.

Park, Jinsoo, Xiang Sun, Jung Il Choi, and Gwang Hoon Rhee. 2017. "Effect of Wind and Buoyancy Interaction on Single-Sided Ventilation in a Building." Journal of Wind Engineering and Industrial Aerodynamics 171 (October). Elsevier Ltd: 380-389. doi:10.1016/j.jweia.2017.10.016.

Ricci, M., L. Patruno, I. Kalkman, S. de Miranda, and B. Blocken. 2018. "Towards LES as a Design Tool: Wind Loads Assessment on a High-Rise Building." Journal of Wind Engineering and Industrial Aerodynamics 180 (July). Elsevier Ltd: 1-18. doi:10.1016/j.jweia.2018.07.009.

Shirzadi, Mohammadreza, Mohammad Naghashzadegan, and Parham A. Mirzaei. 2018. "Improving the CFD Modelling of Cross-Ventilation in Highly-Packed Urban Areas." Sustainable Cities and Society 37 (September 2017). Elsevier: 451-465. doi:10.1016/j.scs.2017.11.020.

Yoder, D. A., J. R. DeBonis, and N. J. Georgiadis. 2015. "Modeling of Turbulent Free Shear Flows." Computers and Fluids 117. Elsevier Ltd: 212-232. doi:10.1016/j.compfluid.2015.05.009. 